CERN-PH-EP/2005-036

26 June 2005

\title{
Reference bars for the alignment of the ATLAS muon spectrometer
}

\author{
Ch. Amelung ${ }^{1)}$, J.R. Bensinger ${ }^{2)}$, F. Cerutti ${ }^{1)}$, C. W. Fabjan ${ }^{1)}$, K. Hashemi ${ }^{2)}$, \\ S. Palestini ${ }^{1)}$, J. Rothberg ${ }^{3)}$, A. Schricker ${ }^{1)}$, I. Trigger ${ }^{1)}$
}

\begin{abstract}
The ATLAS forward, or endcap, muon spectrometer covers the rapidity region of $1.0<|\eta|<$ 2.8 and extends over a volume of approximately two times $5000 \mathrm{~m}^{3}$. High-precision muon tracking chambers are used to measure track sagittas with $50 \mu \mathrm{m}$ accuracy. An optical alignment system will monitor the relative spatial positions of these chambers with an accuracy of $30 \mu \mathrm{m}$, such that the contribution of the chamber location to the global sagitta error remains below $40 \mu \mathrm{m}$. The alignment concept relies on devices called alignment bars; these are long tubes (up to $9.6 \mathrm{~m}$ ) whose shape is constantly monitored at the level of $20 \mu \mathrm{m}$ by internal alignment sensors, and on which further alignment sensors are mounted in order to determine the positions of the nearby chambers. In order to derive the shape of an alignment bar from the readings of the internal sensors monitoring it, a model to describe deformations and a calibration procedure are required. This paper describes the design of the alignment bars, the method for reconstructing the shape from the sensor readings, and the calibration of an alignment bar on a large coordinate-measurement machine. The performance of a single bar, and the performance of an ensemble of bars in a large-scale test environment are discussed.
\end{abstract}

Accepted by Nuclear Instruments and Methods

1) CERN, Geneva, Switzerland

2) Brandeis University, Waltham MA, USA

3) University of Washington, Seattle WA, USA 


\section{Introduction}

Lepton precision spectroscopy is one of the essential detection requirements for the generalpurpose proton-proton experiments at the LHC. Typical performance requirements, for example, for Higgs or SUSY physics, are large rapidity ${ }^{1)}$ coverage, $|\eta|<3$, combined with very good momentum resolution, typically $\Delta p / p \approx 0.1$ at $1 \mathrm{TeV}$. Not surprisingly, the two LHC experiments, ATLAS and CMS, are conceived around these requirements.

The ATLAS muon spectrometer [1] achieves this performance by pushing three major components to the limits of today's detector technologies:

- A set of three, large, superconducting, air-core toroid magnets; one such magnet covers the central, or barrel, region with $|\eta|<1.0$, providing a magnetic field of about $0.5 \mathrm{~T}$; the other two magnets cover the forward, or endcap, regions, $1<|\eta|<2.8$.

- Novel precision tracking chambers ('Monitored Drift Tubes', MDTs) providing $50 \mu \mathrm{m}$ spatial resolution per tracking station, at the highest luminosities and particle rates.

- Novel optical alignment systems for relative alignment of the tracking stations with an accuracy of $30 \mu \mathrm{m}$.

The central toroid is built from eight individual interconnected coils, leaving the major part unobstructed for muons. This open construction also allows the passage of optical rays, which are used to optically connect the muon chambers in successive layers in a quasi-projective geometry, each set of chambers forming a 'tower' pointing towards the pp interaction region.

The alignment in the endcap region, the subject of this paper, could not follow the same alignment strategy. For technical reasons, the eight toroidal coils of one endcap magnet are enclosed in a common cryostat, obstructing the optical path between the chambers in front of and behind the magnet. The concept of projective towers could therefore not be adopted. It was replaced by a system based on a grid of devices called alignment bars.

The basic idea is shown in Figs. 1 and 2. Each bar is equipped with alignment sensors to allow optical connection between the corresponding bars in three planes; it also carries sensors to monitor the chambers in a plane relative to the neighbouring bars. The bars themselves are equipped with internal optical and thermal sensors such that the shape of the bar and the position of all the sensors on the bar are known with adequate accuracy. The length of the bars varies from $1.9 \mathrm{~m}$ in the first plane to $9.6 \mathrm{~m}$ in the two outer planes. The result of this arrangement has the following advantages:

- Each bar can be measured ('calibrated') in the laboratory very precisely on a large 3-D coordinate-measurement machine (CMM); subsequently, the positions of the various sensors on a bar are known within better than $10 \mu \mathrm{m}$. The instrumentation of the alignment bars is used afterwards to monitor deviations (due to mechanical deformation and thermal expansion) from the calibrated geometry during spectrometer operation.

- Each bar serves as a highly precise 'reference ruler', allowing one to establish a grid of absolutely known positions in space.

- The chamber positions are monitored relative to the bars and thus anchored to the reference grid.

In the following we describe the theory and the practical implementation of the alignment bars; we present the concept of the alignment procedure and discuss the results of the full-size test of one octant equipped with alignment bars and tracking chambers, demonstrating that the design performance can be achieved.

1) The pseudorapidity, $\eta$, is defined as a function of the polar angle, $\theta$, which is measured with respect to the beam line: $\eta=-\ln (\tan (\theta / 2))$. 


\section{Requirements and specifications}

The performance specifications of the muon spectrometer require that the positions of the muon chambers be known very precisely. This is achieved by the endcap alignment system in two steps. The chamber positions are related by proximity monitors (CCD cameras looking at a coded RASNIK [2] mask) to the nearest alignment bars, and the positions of the bars with respect to each other are reconstructed from measurements of the polar and azimuthal monitors ('BCAMs', Boston CCD Angular Monitor [3], CCD cameras looking at laser diodes).

The alignment bars with their optical alignment instrumentation have the role of providing a precisely monitored 3-D alignment grid against which all the muon tracking chambers are optically referenced. The bars are internally instrumented so as to allow the reconstruction of the bar shape and alignment sensor positions at the $20 \mu \mathrm{m}$ level. The bars carry alignment sensors for two different types of measurements:

- BCAMs connect alignment bars along azimuthal (within a chamber plane) and polar (between different chamber planes) lines, thus establishing the 3-D coordinate system. BCAMs have a an absolute angular accuracy of $50 \mu \mathrm{rad}$, and a relative angular resolution along the polar lines of $5 \mu \mathrm{rad}$.

- Proximity sensors monitor the chambers relative to the bars, with an absolute accuracy of $20 \mu \mathrm{m}$. The alignment bars carry the RASNIK masks for these sensors, and the corresponding cameras are mounted on the chambers.

\section{Alignment bar design}

\subsection{Design concept}

The main concept of the alignment bar design is to use a free body on which no external forces (apart from those compensating gravity, of course) are acting; this allows the positions of the various sensors on the bar to be related in a controllable manner. Consequently, unknown external forces acting on the bar need to be avoided as much as possible, in order to permit the reconstruction of its shape with the knowledge of only a few sampling points. Shape changes perpendicular to the bar can be monitored with optical methods, changes in length are indirectly derived from changes in temperature. A view of a section of an alignment bar is shown in Fig. 3.

\subsection{Mechanical description}

One overriding requirement was that all the instrumentation to monitor the length and shape of the alignment bar should be placed inside it. This ensures that all components are well protected, and that no parts of this instrumentation block space for the sensor platforms mounted on the outside of the bar. Commercially available cylindrical aluminium tubes (Anticorodal 6004) with $80 / 85 \mathrm{~mm}$ outer and $72 / 77 \mathrm{~mm}$ inner diameter have been chosen. ${ }^{2)}$ The use of a cylindrical tube has the advantage that all the sensor platforms mounted on the bar can be aligned with an angular precision of $1 \mathrm{mrad}$. In addition, the resistance against deformation is isotropic, which reduces uncertainties in predicting the shape.

Alignment bars are kinematically supported at two points. The position of these support points along the bar is chosen such that the overall deformation due to gravity is minimized. A kinematic mount of a free body has to be designed such that all six degrees of freedom are constrained, to ensure that the bar does not move, while at the same time no stress is introduced by overconstraining any degree of freedom. Consequently the constraint forces, namely those

2) The option of carbon fibre bars was considered, but discarded because of their dimensional changes with changes of humidity. 
external forces required to maintain the position of the free body, can be calculated from the six conditions of equilibrium,

$$
\sum_{i=1}^{n} F_{i}=0, \quad \sum_{i=1}^{n} \Gamma_{i}=0,
$$

where $F_{i}$ and $\Gamma_{i}$ are the external forces and torques acting on the free body. Mounts where every motion is decoupled are expensive and complex, thus one of the bar mounts has been chosen as a simplified loose bearing (Fig. 4). This bearing exerts torque due to forces induced by the weight of the bar not acting in the centre of symmetry if the two bearing angles $\alpha$ and $\beta$ are non-zero (Fig. 5). The influence of these torques on the shape reconstruction is negligible [4].

\subsection{Bar shape sensors}

The first- and second-mode deformations of a kinematically supported bar can be detected by measuring the deformations at a minimum number of five points along the bar. Optical straightness monitors (RASNIKs) fulfil the requirement of measuring deviations from a straight line to a few microns over a distance of $10 \mathrm{~m}$; one RASNIK system, composed of CCD, lens, and mask, can measure displacements of one of the elements relative to the two others. In order to measure five points relative to each other, two more RASNIK systems have to be added. Figure 6 shows this concept of three overlapping RASNIK systems. The three systems are coupled by mounting two elements of different RASNIKs on the same support. After having gained some experience with the system of three RASNIK lines, it was subsequently extended to four lines for the ATLAS alignment bars (Fig. 6). This modification dramatically increases the dynamic range in terms of bar deformation, and the system also gains redundancy. In terms of reconstruction accuracy, both systems are equivalent. We shall discuss only the three-RASNIK bar in this paper; the extension to the four-RASNIK bar is straightforward.

Figure 7 shows how to interpret the RASNIK measurements. Deviations on the RASNIK mask from the 'zero-reading' provide the position information of three points (CCD, lens and mask of the long RASNIK) relative to the straight line defined by the positions of the lenses of the short RASNIKs. Each RASNIK system measures the following quantity (in the two coordinates transverse to the optical axis):

$$
x_{\mathrm{RAS}}=\left(1+m^{-1}\right)\left(x_{\text {lens }}-x_{\mathrm{CCD}}\right)-\left(x_{\text {mask }}-x_{\mathrm{CCD}}\right),
$$

where the magnification $m$ represents the ratio of the distances between the RASNIK elements along the optical path:

$$
m=\frac{z_{\mathrm{CCD}}-z_{\text {lens }}}{z_{\text {lens }}-z_{\text {mask }}} .
$$

The equations can be coupled through the elements that are mounted on the same support:

$$
x_{\mathrm{CCD}, 1}=x_{\mathrm{CCD}, 3}, \quad x_{\text {mask }, 1}=x_{\text {mask }, 2}=x_{\text {lens }, 3}, \quad x_{\mathrm{CCD}, 2}=x_{\text {mask }, 3} .
$$

\subsection{Temperature sensors}

The alignment sensor positions on an alignment bar have to be known with a longitudinal accuracy of $20 \mu \mathrm{m}$; the positions of the sensors change during operation because of the thermal expansion of the aluminium bars. This problem is easily solved by measuring the bar temperature at several points along the bar and deriving the changes in length from the temperature profile.

The thermal expansion of the alignment bar can be described as:

$$
\delta L(T)=\alpha \int_{0}^{l}\left(T(z)-T_{0}\right) d z
$$

where $\alpha=23.8 \mu \mathrm{m} \mathrm{K}^{-1} \mathrm{~m}^{-1}$ is the thermal expansion coefficient of the bar material, and $T_{0}$ is the temperature at the length $L_{0}$. As the bar temperature is measured at discrete points along 
the bar, the simplest way of calculating the bar length is to reduce equation (5) to:

$$
\delta L(T)=\alpha \sum_{i=1}^{n-1}\left(\bar{T}_{i}-T_{0}\right) l_{i}, \quad n=\text { number of sensors. }
$$

Here, $\bar{T}_{i}$ is the average temperature of two adjacent sensors, $\left(T_{i}+T_{i+1}\right) / 2, l_{i}$ is the distance between two sensors. In the same way as for RASNIK measurements, deviations of the measured temperatures from a 'zero-reading' are used to track changes in the bar length.

\subsection{In-bar instrumentation support structure}

The components of the optical sensors and the temperature sensors are mounted on disks. These are connected to each other by a skeleton-like structure, which is inserted into the alignment bar from one end. The bar heads containing the electronics for the in-bar instrumentation are attached at the tube ends.

The structure shown in Fig. 8 is designed such that all the in-bar sensors can be mounted and connected while the skeleton is outside the bar. After the insertion of the structure into the tube, only the disks are rigidly attached to the bar, with one screw per disk, thus aligning them to the inner wall of the tube. This minimizes the effect on the mechanical properties of the bar. The temperature sensors are put in thermal contact with the tube by small springs.

\section{Analytic bar shape model}

The in-bar instrumentation provides a precise measurement of the positions of three points on the bar, relative to two other points on the bar that define the $Z$ axis of the bar coordinate system. Between these five points, an analytic model of the bar shape is needed for interpolation.

The ideal alignment bar can be considered as a straight tube, perfect in circularity with a constant cross-section along its axis. The deformation of such an ideal bar, when exposed to external forces, can be calculated analytically under the assumption that cross-sections of the bar remain planar, and perpendicular to the neutral line of the bar, independent of its deformation. This assumption is justified for large objects and provides exact solutions for simple bending. Assuming a large object where the product of Young's modulus, $E$, and the second moment of inertia, $I$, remains constant, the deformation $w(z)$ due to external forces can be written as:

$$
E I \frac{d^{4}}{d z^{4}} w(z)=q(z)
$$

where $q(z)$ represents the load distribution. Equation (7) is a linear differential equation, thus its solutions can be superimposed. Consequently, the different contributions to the deformation of the alignment bar can be calculated separately: the deformation due to the presence of discrete forces (like alignment sensors and platforms), and the deformation due to a continuous load distribution (the weight of the bar itself).

As an example, we discuss here the solution of the above differential equation for discrete forces: the most general case that needs to be considered is a tube supported in two points and exposed to three external forces, as illustrated in Fig. 9. The calculations can then easily be extended to an arbitrary number of forces by superimposing results for three external forces as many times as required.

Figure 9 shows that five forces in total deform the bar: three external forces $\left(F_{1}, F_{3}, F_{5}\right)$, and the two forces $\left(F_{2}, F_{4}\right)$ acting in the support points. The two latter forces can be calculated using the kinematic conditions of equilibrium, i.e. the sum of all torques as well as the sum of all forces has to be zero:

$$
\sum_{n} \Gamma_{n}=0, \quad \sum_{n} F_{n}=0, \quad n \in[1, \ldots 5]
$$


To calculate the deformation of the bar, equation (7) has to be applied to each section between two adjacent forces. Hence the six resulting equations can be written as:

$$
E I \frac{d^{3}}{d z^{3}} w_{i}(z)=\sum_{n=0}^{i} F_{n}\left(z_{n}\right) \quad i \in[0, \ldots 5],
$$

where $i$ denotes one of the six sections of the bar, and $F_{0}=0$. The equations (9) are coupled using the following static boundary conditions:

$$
\begin{aligned}
w_{2}\left(z_{2}\right) & =0 \\
w_{4}\left(z_{4}\right) & =0 \\
w_{i}\left(z_{i}\right) & =w_{i+1}\left(z_{i}\right), \\
\frac{d}{d z} w_{i}\left(z_{i}\right) & =\frac{d}{d z} w_{i+1}\left(z_{i}\right) .
\end{aligned}
$$

Since no external torques are acting on the system, the remaining kinematic boundary conditions can be written as:

$$
\begin{aligned}
\frac{d^{2}}{d z^{2}} w_{0}(0) & =0 \\
\frac{d^{2}}{d z^{2}} w_{5}(l) & =0 \\
\frac{d^{2}}{d z^{2}} w_{i}\left(z_{i}\right) & =\frac{d^{2}}{d z^{2}} w_{i+1}\left(z_{i}\right) .
\end{aligned}
$$

The solutions of the differential equations (9) are six polynomials (one for each interval) of at most third order.

\section{Bar shape reconstruction}

The reconstruction of the full alignment bar shape proceeds in two steps, making use of the analytic bar shape model. In the first step, the effect of all known forces acting on the bar (sensor weights, effective change in gravity due to inclination of the bar) is computed and compared to the RASNIK measurements. The difference between computed and measured RASNIK values is used in the second step to determine three effective forces assumed to be acting on the bar in addition to the known ones. ${ }^{3)}$ The effective forces absorb everything that was not accounted for in the first step, like neglected forces (weights of cables), and uncertainties of parameters (weight of the bar tube and the in-bar instrumentation, Young's modulus and geometrical moment of inertia of the tube). The quality of the interpolation depends on the effective forces being small, as a systematic error is made in absorbing all unaccounted-for effects into them. This error must not exceed the required uncertainty of the bar shape of $20 \mu \mathrm{m}$, and consequently, the bar shape predicted in the first interpolation step should be as accurate as possible.

\section{Calibration of alignment bars}

The tubes for the alignment bars as delivered by the manufacturer have an undefined shape. Their straightness is specified to be better than $1 \mathrm{~mm} / \mathrm{m}$, two orders of magnitude worse than the level of accuracy needed. In order to use a shape model to describe the bar shape (which assumes an initially straight bar), the initial shape of the bar needs to be known; after that, any deformations are well described by the model, as they are superimposed linearly to the initial shape. To derive any change in shape of the alignment bar from the readings of its in-bar

3) The effective forces may be chosen as either discrete forces or continuous load distributions. While there is no difference between the two in principle, the latter has some advantages for practical application. 
instrumentation, a calibration procedure is required. The bar calibration must therefore provide two sets of data:

- an initial shape of the bar, and

- an in-bar sensor calibration, i.e. a prescription of how to modify the initial shape when the readout values of the in-bar sensors change.

The initial bar shape can be understood as represented by the positions of all points mechanically connected to the bar that are relevant later in the alignment; the shape of the alignment tube itself is completely irrelevant. Each alignment sensor (BCAM, RASNIK mask) is kinematically mounted on three steel balls glued onto a platform on the alignment bar. Thus the initial shape measurement is obtained by measuring with a CMM the positions of all these balls in the bar coordinate system.

The calibration of the in-bar sensors relates the three individual RASNIK coordinate systems (defined by CCD, lens, and mask) to one common coordinate system, which is the bar coordinate system in which the ball positions were measured. The calibration of one in-bar RASNIK proceeds as follows:

- Auxiliary balls are glued on the bar surface, in approximately those places where the RASNIK elements are located inside the bar.

- The positions of all balls on the bar are measured with the CMM. In parallel the RASNIKs are read out.

- The bar is deformed such that the readout values of the RASNIKs change significantly. The ball positions are re-measured and the corresponding RASNIK values are recorded.

In order to obtain the relation between changes in ball positions and in RASNIK values, the quantities from equation (2) are calculated from the measured positions of the auxiliary balls, and are compared to the RASNIK values. Two free parameters, a scaling factor (very close to unity) and a rotation of the RASNIK coordinate system relative to the bar coordinate system, are sufficient to make them agree.

\section{Performance of a single alignment bar}

Six alignment bars have been built for the H8 test setup at CERN, which is described in more detail in Section 8. Tests related to the thermal expansion of the bars were performed at CERN, after which all the bars were temporarily shipped to Freiburg University (Germany) in order to calibrate the in-bar instrumentation and to measure the initial shape with the large CMM available there.

\subsection{Bar length reconstruction}

In order to compare the bar length as derived from temperature sensor measurements to a direct length measurement, the first alignment bar was equipped with an internal quartz rod, guided by the tube connecting the inner stations and supported by springs placed in various positions along this tube. The expansion of the bar relative to the expansion of the quartz rod was measured with a capacitive sensor providing relative distances with an accuracy of $1 \mu \mathrm{m}$.

To study the bar length measurement in an ambient temperature environment, day-night temperature variations were used. It turned out that measuring the expansion of the alignment bar relative to the quartz rod is affected by a systematic error: stress induced by friction at the points where the rod is supported causes the rod to expand or contract, depending on the temperature trend. Figure 10 shows the measured hysteresis, which is of the order of $20 \mu \mathrm{m}$. 
The force required to explain this hysteresis agrees well with the force that was measured by pulling the quartz rod out of the tube with a spring balance. After correcting for this effect, an error in length prediction of $3.4 \mu \mathrm{m}$ r.m.s. is obtained for an ambient temperature ranging from $18^{\circ} \mathrm{C}$ to $27^{\circ} \mathrm{C}$. A second test was performed using a laser interferometer as reference. Here, a residual error in deriving the bar length from its temperature of $1.5 \mu \mathrm{m}$ r.m.s. was obtained for temperatures between $21.5^{\circ} \mathrm{C}$ and $25.5^{\circ} \mathrm{C}$.

The measurements with the quartz rod and the laser interferometer were also used to determine the expansion coefficient of the alignment bar, which was found to be $\alpha=22.0 \pm$ $0.3 \mu \mathrm{m} \mathrm{K}^{-1} \mathrm{~m}^{-1}$ (significantly less than the value provided by the manufacturer of the aluminium tube).

Based on these results, we concluded that the temperature-induced changes of the alignment bars could be adequately described by knowing the temperature distribution along the bars. Therefore, all future bars were instrumented with 27 Pt-100 sensors distributed along the bar.

\subsection{Bar shape reconstruction}

The bar shape reconstruction procedure was tested using CMM measurements of a single alignment bar. The forces acting on a bar scale with gravity, thus the most interesting test in terms of predicting the bar shape using the analytic model is to measure an alignment bar in the horizontal position (referred to below as $0^{\circ}$, i.e. the initial shape measurement), to rotate the bar around its axis by $180^{\circ}$, and to compare the calculated shape to the shape measured in this orientation. This procedure will be discussed here. In terms of forces acting on the bar it means that gravity is inverted in the vertical plane, and thus represents a worst-case scenario for the ATLAS alignment bars.

All CMM measurements were performed in a bar coordinate system defined by three balls glued to the bar. The $9.6 \mathrm{~m}$ long bars had to be measured in two portions, $0-6200 \mathrm{~mm}$ and $3400-9600 \mathrm{~mm}$, because of the limited reach of the CMM. The three points in the overlap region defining the coordinate system of the bar were then used to combine the two measured portions into one, for each measured orientation.

When rotating the bar by $180^{\circ}$, the deformation of the bar mainly takes place in the coordinate along gravity $(Y)$, reaching $6 \mathrm{~mm}$ at one of the ends for the bar under study (this depends of course on the mass distribution along the bar). A small deformation of about $100 \mu \mathrm{m}$ r.m.s. in the other coordinate $(X)$ is observed as well and is ascribed to the fact that the bar coordinate system was not perfectly aligned to the direction of gravity. This 'misalignment' of the coordinate system is negligible and can be easily absorbed into effective forces, thus assuming for simplicity that gravity has no component along $X$.

By taking into account the effect of all known forces acting on the bar (which, in this case, means to add twice the gravitational sag of the bar tube and the effect of all the platforms), and superimposing the final correction of three effective forces derived from RASNIK measurements, a prediction for the $180^{\circ}$ measurement is derived from the $0^{\circ}$ measurement. The difference between the predicted and the measured ball positions is shown in Fig. 11; they are found to agree within 10-20 $\mathrm{m}$ r.m.s. and the large deformation along the direction of gravity is reconstructed essentially as well as the tiny deformation transverse to gravity, which supports the confidence in the shape reconstruction method.

From the results presented in this section, we conclude that the analytic bar shape model is adequate to describe alignment bar deformations to better than $20 \mu \mathrm{m}$.

\section{Test beam results}

\subsection{Setup and software}

The ATLAS muon collaboration installed a large-scale test setup of the muon spectrometer barrel and endcap in the $\mathrm{H} 8$ test beam line at CERN (for the results presented here, no use was 
actually made of the beam). The endcap part of the setup consists of three pairs of large and small MDT chambers, arranged such that they approximately represent a part of an octant of the EI (inner), EM (middle), and EO (outer) stations of the ATLAS muon endcap. In addition, there are six alignment bars (two $2.6 \mathrm{~m}$ short ones in the EI station, and four $9.6 \mathrm{~m}$ long ones in the EM and EO stations). Chambers and bars are equipped with a full set of alignment and temperature sensors. This is the smallest muon endcap system unit that can be aligned and used for track reconstruction. The chamber positions have been chosen such that the muon beam from the SPS illuminates mainly the large chambers, traversing them at $15^{\circ}$. Chambers and bars are mounted kinematically on large support structures of aluminium and steel, which have been designed to be conceptually similar to the corresponding structures in ATLAS. A detailed description of the setup and of test results will be given in [5]; the following sections describe in detail only tests that are related to the alignment system.

The program ARAMyS [6] has been used to reconstruct from the alignment sensor measurements the geometry of the setup, i.e. the deformations of individual chambers and alignment bars, and their relative positions and orientations. ARAMyS is based on a universal concept of alignment and treats in a unified way information from sources as different as, for example, CCD cameras, temperature sensors, and results from external surveys. The alignment is reconstructed by comparing measured values from the sensors to expected values for an assumed set of local coordinate system positions and orientations, as well as chamber and alignment bar deformations, and by iteratively minimizing the difference (defined as a $\chi^{2}$ in the usual way) by variations of these assumed sets of numbers using MINUIT [7].

\subsection{Measurements on single alignment bars}

As described before, alignment bars are calibrated in the horizontal position on a CMM. At this time, they are equipped with temperature sensors and in-bar RASNIKs; however, BCAMs, proximity sensor masks, and cables are mounted only after installing the bars in their final location, and deform the bars along the direction of gravity. In addition, only three bars in the H8 setup are approximately horizontal; the other three are inclined by about $45^{\circ}$, and thus deform because the component of gravity transverse to the bar axis is decreased (in ATLAS, all alignment bars are inclined, by angles between $14^{\circ}$ and $76^{\circ}$ with respect to the horizontal direction).

For an accurate bar shape reconstruction, the bar shape predicted in the first interpolation step should be as accurate as possible. This has been studied with the four long alignment bars in the setup. In Fig. 12, the computed and the observed bar shapes are compared for two of them. This comparison is strictly valid only at the five points where RASNIK elements are placed; in between them an approximate interpolation is shown for illustration. It is found that for all four bars the computed shape agrees with the observed one to better than $200 \mu \mathrm{m}$ r.m.s. Consequently, in order to obtain the real bar shape to better than $20 \mu \mathrm{m}$, the error of the interpolation procedure must not exceed 10\%. This was verified before using CMM measurements (Section 7.2). In addition, an indirect check can be performed using the test setup, by verifying the internal consistency of measurements from BCAMs mounted on the bars; this is the subject of the following section.

Short alignment bars deform significantly less than long bars, by 1-2 orders of magnitude. Their total deformations stay below $30 \mu \mathrm{m}$, so these bars could almost be regarded as rigid bodies (except for thermal expansion). Consequently, the RASNIKs monitoring the bars are in principle dispensable for the purpose of measuring deformations; their main purpose is rather to ensure that the bars are not accidentally exposed to large and otherwise unnoticed forces.

A slightly surprising observation made at the test beam setup was temperature-related shape variations of the long alignment bars (Fig. 12). Bars should not deform transverse to the bar axis on account of thermal expansion (acting only along the bar axis in a significant way), because the bar mounts are designed such that their positions along the bar (expressed as fractions of the varying bar length) do not change, and the effect of longer bars sagging more 
under their own weight is negligible $\left(0.2 \mu \mathrm{m}\right.$ for a temperature change of $\left.1^{\circ} \mathrm{C}\right)$. It turns out that the variations are correlated to the derivative of temperature with time: the bars deform more when expanding faster, and the sign of the deformation is different for increasing and decreasing temperatures. This suggests that friction in the sliding bar mount (the loose bearing) is responsible for the effect, i.e. that during thermal expansion the movement of the bar in the mount ${ }^{4)}$ is not completely free because of the frictional force acting on it longitudinally, below its centre. Apart from being an interesting effect, the magnitude and speed of the variations of less than $50 \mu \mathrm{m}$ over many hours are too small to affect the performance of the alignment system; still, an attempt was made to reduce friction in the final ATLAS version of the sliding mount by a modification of the surface treatment.

\subsection{Measurements with the grid of six alignment bars}

There are two strategies for making use of the data provided by an alignment system. The absolute concept is the straightforward one: to ask that the alignment system provides chamber positions at any time and without using any external references. The other concept is relative: assuming that at one moment the chamber positions are known (e.g. from the use of straight muon tracks in special runs with the magnetic field switched off, or using muons from $\mathrm{Z}^{0}$ decays recorded in normal running), the alignment system is used only to follow variations of the positions from this point on. The advantage of the latter strategy is that all sensor positioning accuracies and many sensor calibration parameters cancel out to first order. Since not all sensors were calibrated at the time of these tests, tests of the absolute concept were performed with the system of alignment bars and BCAMs (described in this section), and tests of the relative concept were performed with the full system of bars and muon chambers (next section).

For tests using the full grid of six alignment bars, it is useful to have an external reference to which the bar positions determined by the alignment system can be compared. An optical survey, with an accuracy of $300 \mu \mathrm{m}$ in each coordinate, was chosen as the reference. Alignment data were taken in parallel to the survey (and averaged afterwards). Alignment bars have two survey rings near the mounts, each of which can hold three survey targets; on average, about two targets per ring could be both installed and seen by the surveyors.

For the alignment reconstruction, the alignment bars are constrained to the locations determined in the optical survey in such a way that for alignment bars with more targets measured than required to determine the position, the best fit of the location in space is obtained; in addition, the internal consistency of the survey data can be verified, and is found to be well within the specified survey precision of $300 \mu \mathrm{m}$. Having checked this, a combined alignment fit is performed, taking into account the in-bar RASNIK and temperature sensor readings, the BCAM measurements, and the survey measurements. The internal consistency of all the sensor measurements in this fit, assuming their design intrinsic resolutions and mounting accuracies, can be taken as a measure of the compatibility of the alignment system with the optical survey, and of how well the sensors (i.e. the system of bars with BCAMs mounted on them) comply with their specifications. To illustrate the result, pull values are extracted for BCAMs after convergence of the fit, assuming the design errors of the sensors, and separately for three classes of BCAM measurements:

- BCAM measuring the absolute position of one light source on its partner,

- BCAM measuring the relative positions (i.e. the separation) of the two light sources on its partner, and

- polar BCAM measuring the relative positions of two light sources on its two partners.

4) An aluminium bar of $9.6 \mathrm{~m}$ length expands by $214 \mu \mathrm{m}$ for a temperature change of $1^{\circ} \mathrm{C}$. If the temperature changes by $5^{\circ} \mathrm{C}$ in 12 hours, this results in an expansion speed of some hundred atomic layers per second. 
In this procedure, RASNIK and temperature sensor measurements are implicitly assumed to be perfect, thus any errors of these devices are absorbed into those of the BCAMs and the survey.

The resulting BCAM pull distributions are shown in Fig. 13, where they are compared with the corresponding distributions as expected from simulations (in order to verify that correlations between sensors have no significant impact on the shape of the pull distributions, and to take properly into account their effect on the width, as well as the effect of the constraints imposed by the survey). It is found that for absolute BCAM measurements, the obtained pull distributions from the setup are centred around zero, and have a width of about unity, as expected from the simulation. ${ }^{5)}$ The two classes of relative BCAM measurements also have approximately zero mean, but narrower than expected pull distributions; this indicates that, for relative measurements, the system of bars and BCAMs performs even better than required. Pull distributions for the survey data are also found to agree with expectations (not shown). From these test results it is concluded that the system of alignment bars and BCAMs works according to specifications.

\subsection{Measurements with the muon simulator}

Tests of the full alignment system require an external reference with a precision that is one order of magnitude better than that of a survey. One possibility for implementing such an external reference (besides real muon tracks) is a device called a muon simulator. It consists of a camera with the optical axis pointing along the straight line corresponding to a hypothetical muon track, and of light sources on MDT chambers whose positions can be measured. From the observed relative movements of the light sources, the sagitta variations can be directly extracted. As the mounting positions of the camera and of the light sources are not known with high accuracy, this device does not provide an absolute reference and can track only variations of the alignment, i.e. be used to test the relative alignment concept. It provides equally precise reference measurements in the bending direction and along the wires; in this respect the muon simulator is superior to using real muon tracks.

A muon simulator was present in the endcap test setup during most of the beam time. For simplicity it was decided to use BCAMs for the camera as well as for light sources, while a technology 'more different' (ideally even a non-optical one) from that used in the alignment system itself might have been more desirable. Eight BCAM cameras were placed on a tripod, and the BCAM light sources were mounted on extension plates attached to the central cross plates of the large chambers, thus modelling a straight muon track that just missed the chambers by $100-200 \mathrm{~mm}$. Of the eight cameras, five could be adjusted such that all light sources were visible to them. The intrinsic error of the sagitta measurement obtained from averaging over the results from the five cameras could be estimated from the data, and was found to be about $10 \mu \mathrm{m}$.

The comparison of sagitta variations in the bending direction (which is inclined by $14^{\circ}$ with respect to the horizontal direction in the test setup) as reconstructed by the alignment system to those measured by the muon simulator (Fig. 14) yields an r.m.s. accuracy of $14 \mu \mathrm{m}$ over a continuous period of 2.5 days, during which daily temperature-induced sagitta variations of up to $500 \mu \mathrm{m}$ were complemented by artificial variations of up to $5 \mathrm{~mm}$ from shifting and/or rotating different chambers. The total $\chi^{2} /$ ndf of the alignment fit varies typically in an acceptable range of $0.9-1.5$. The observed resolution of the alignment system agrees well with the expectation from simulations for the relative alignment (the sensor mounting accuracies, which do not contribute here, dominate the absolute alignment design resolution of 30-40 $\mu \mathrm{m}$ ). The same comparison in the wire direction (not shown) yields an r.m.s. accuracy of about $125 \mu \mathrm{m}$, safely below the required accuracy of $250 \mu \mathrm{m}$ in this coordinate. From these test results it is concluded that the full alignment system for MDT chambers works according to specifications in the relative

\footnotetext{
5) Measurements from two BCAMs (out of 36 present in the setup) have been excluded from the alignment reconstruction because their measurements deviated largely from the expectation (by $4.5 \sigma$ and $10.5 \sigma$, respectively). The reason for this is unclear, but problems with the CMM measurements of the balls upon which these BCAMs are mounted are the most likely cause.
} 
alignment mode; the test in the absolute alignment mode requires a full set of calibrations and will be performed using data collected later in 2004 .

\section{Summary}

The alignment concept of the ATLAS muon spectrometer endcap relies on devices called alignment bars to localize the muon chambers with a sagitta accuracy of $50 \mu \mathrm{m}$, corresponding to a relative momentum resolution of $10 \%$ for a $1 \mathrm{TeV}$ muon. Alignment bars are long tubes (up to $9.6 \mathrm{~m}$ ) whose shape is constantly monitored at the level of $20 \mu \mathrm{m}$ by internal alignment sensors, and on which further alignment sensors are mounted. We have presented the design of the alignment bars, the method to reconstruct the shape from the sensor readings, and the calibration of an alignment bar on a large coordinate-measurement machine. The performance of a single bar, and the performance of an ensemble of bars in a large-scale test environment, were studied extensively, and the design was found to comply with the specifications in all aspects.

\section{Acknowledgements}

The results presented in this paper could not have been obtained without the work of many colleagues not listed as authors, and their contributions are gratefully acknowledged. We would like to express our thanks explicitly to Th. Alexopoulos, P. Oikonomou, and Y. Tsipolitis (NTU Athens) who set up the alignment bar temperature readout system, to K. Handrich and J. Tobias (Freiburg University) who performed the CMM measurements for the alignment bar calibration, to the colleagues from MPI Munich who contributed to the design of the gimbal mounts, and to the many people who contributed to the big effort of setting up and running the H8 system test of the muon spectrometer. The installation of chambers and alignment bars and most of the setup in the beam line would have been nearly impossible without the dedicated and efficient work of W. Andreazza (CERN).

\section{References}

[1] ATLAS Muon Collaboration, ATLAS Muon Spectrometer Technical Design Report, CERN/LHCC/97-22.

[2] H. Dekker et al., The RASNIK/CCD 3-dimensional alignment system, Proceedings of the Third International Workshop on Accelerator Alignment, CERN, Geneva (1993).

[3] K. Hashemi and J. Bensinger, The BCAM Camera, ATLAS note ATL-MUON-2000-024.

[4] A. Schricker, The Alignment System of the ATLAS Muon Endcap Spectrometer, Ph.D. thesis, Vienna University of Technology (2002), available from http://atlas.web.cern.ch/Atlas/documentation/thesis/schricker/thesis.pdf.

[5] ATLAS Muon Collaboration, System test of the ATLAS muon spectrometer in the H8 test beam area at CERN, to be submitted to NIM.

[6] Ch. Amelung, Alignment Reconstruction and Simulation for the ATLAS Muon Spectrometer: ARAMyS Manual, available from http://cern.ch/amelung/aramys.html.

[7] F. James and M. Roos, Comput. Phys. Commun. 10 (1975) 343; MINUIT Reference Manual. 


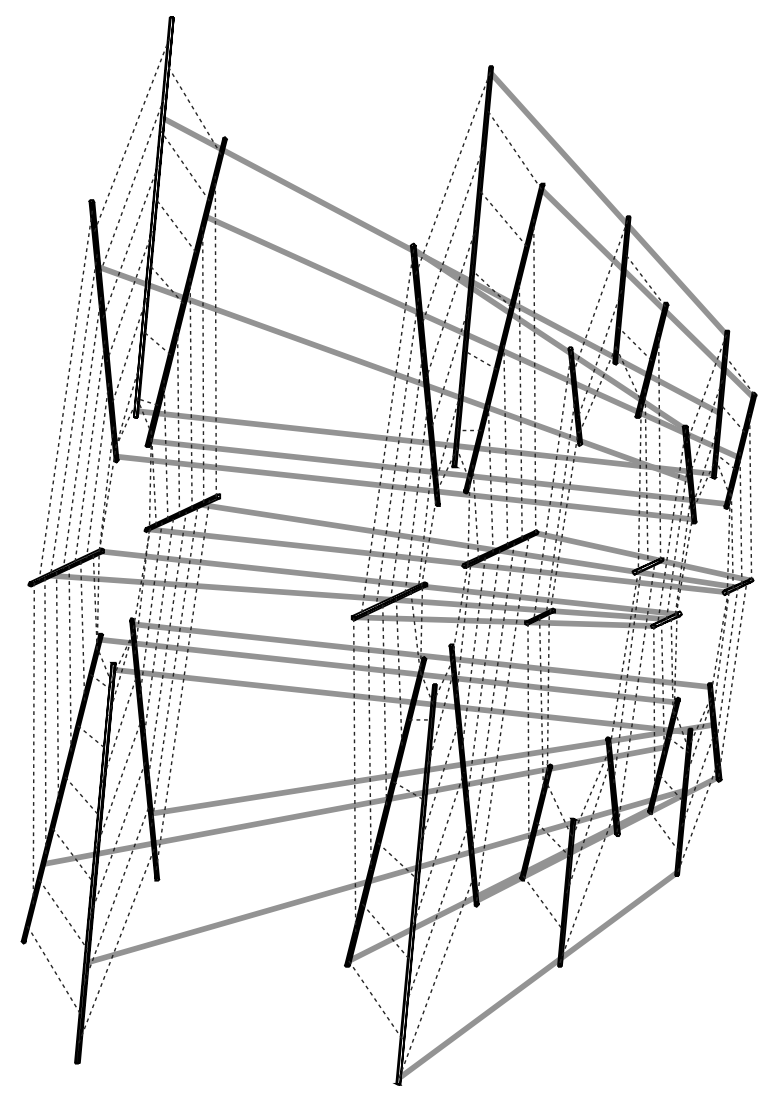

Figure 1: Alignment bars and alignment rays in one endcap of the ATLAS muon spectrometer. For clarity of presentation, the MDT chambers are not shown; they are arranged in four planes, each corresponding to a set of eight alignment bars in the figure. The ATLAS interaction point is on the right; depending on the angular range, muons are detected in the first (EI), second (EE), and third plane (EM), or in the first, third, and fourth (EO) plane.

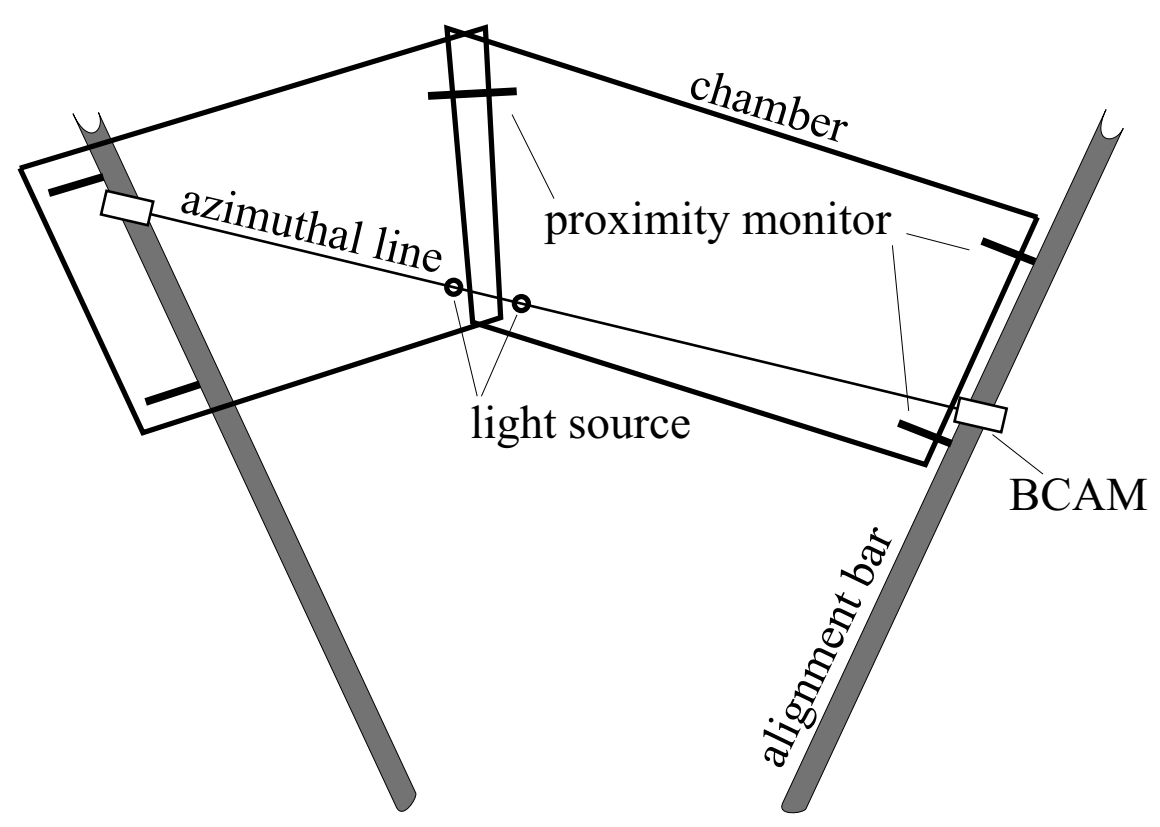

Figure 2: Two alignment bars and a small-large MDT chamber pair in one plane. 


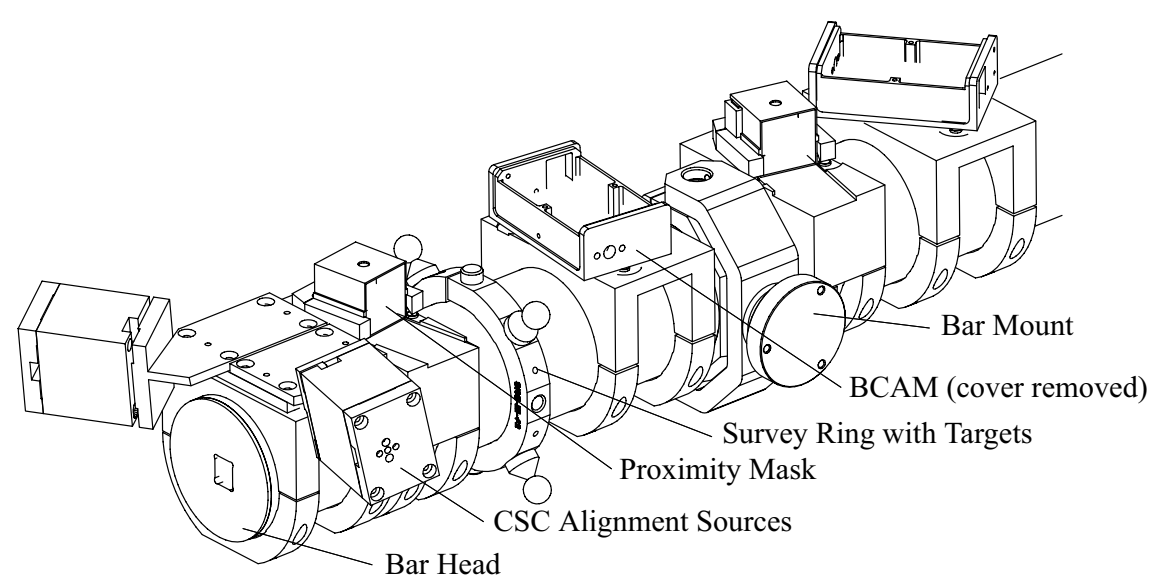

Figure 3: Detailed view of an alignment bar, with various sensors mounted onto it.
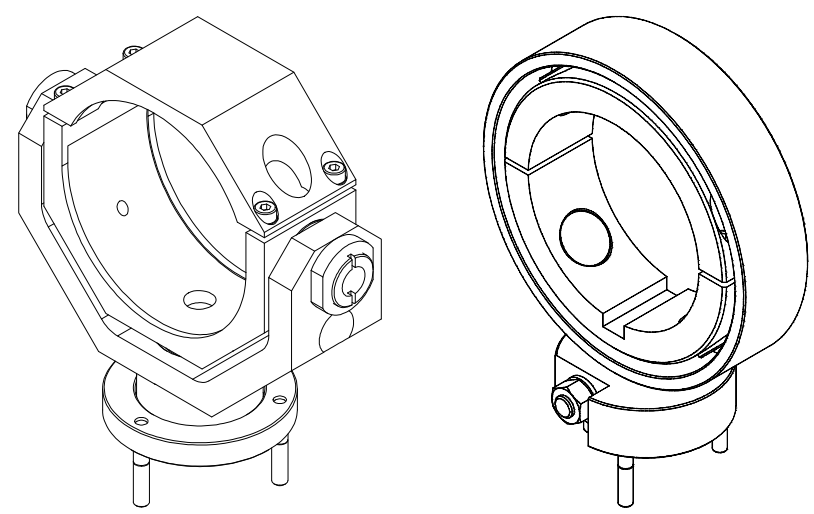

Figure 4: Left: gimbal mount permitting two degrees of freedom (rotation around the $X$ and $Y$ axes); Right: simplified loose bearing permitting four degrees of freedom (rotation around the $X, Y$, and $Z$ axes, and linear motion in the $Z$ direction).

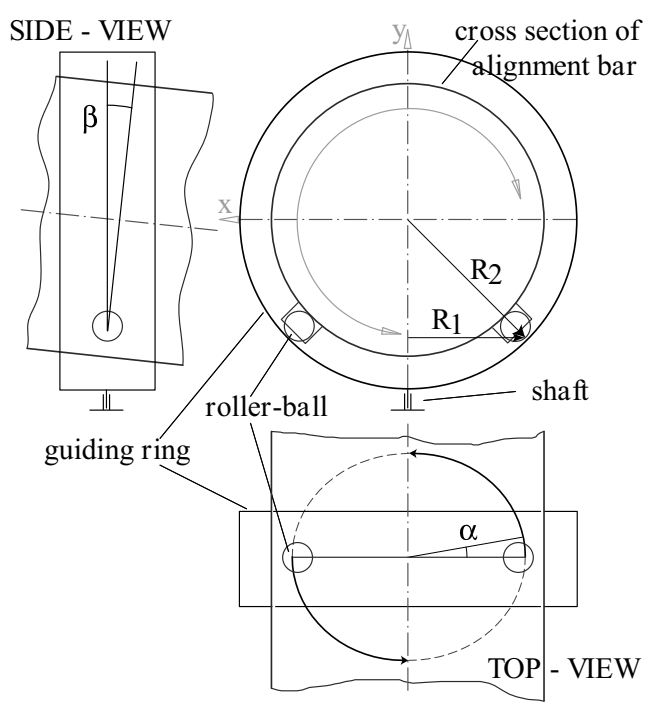

Figure 5: Illustration of the torque exerted by the simplified loose bearing owing to forces induced by the weight of the bar. 

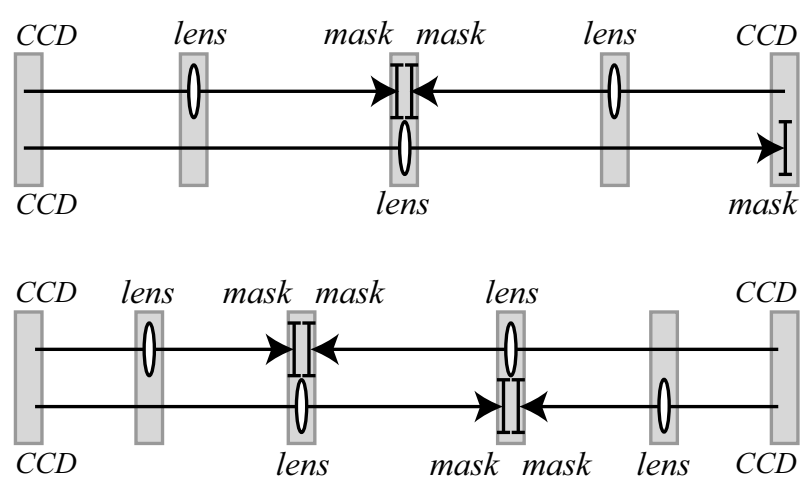

Figure 6: Two different layouts of the optical in-bar instrumentation; Top: three overlapping RASNIK systems; Bottom: four overlapping RASNIK systems.

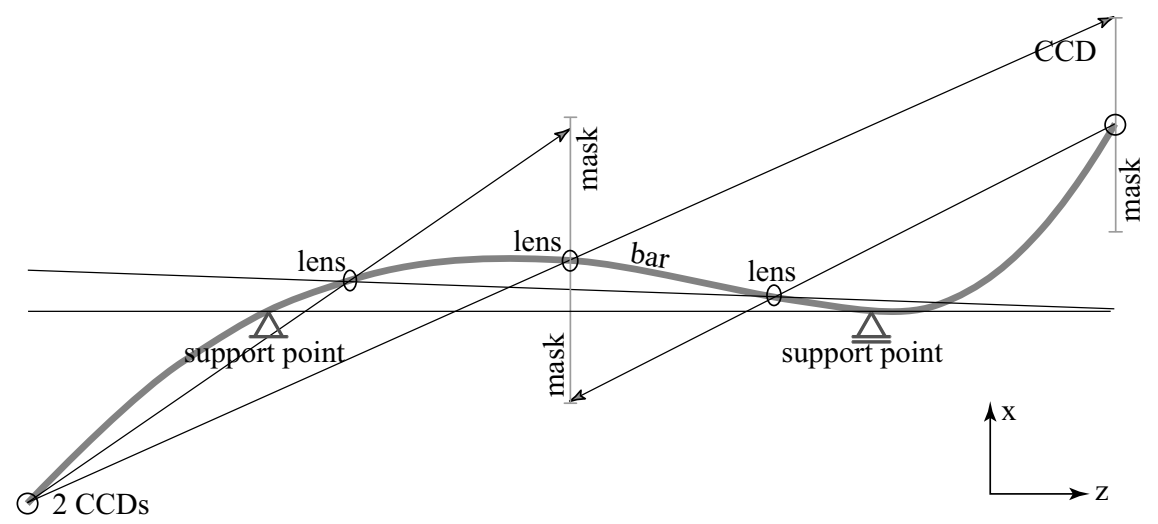

Figure 7: Interpretation of the measurements provided by the optical in-bar instrumentation (for three RASNIKs).

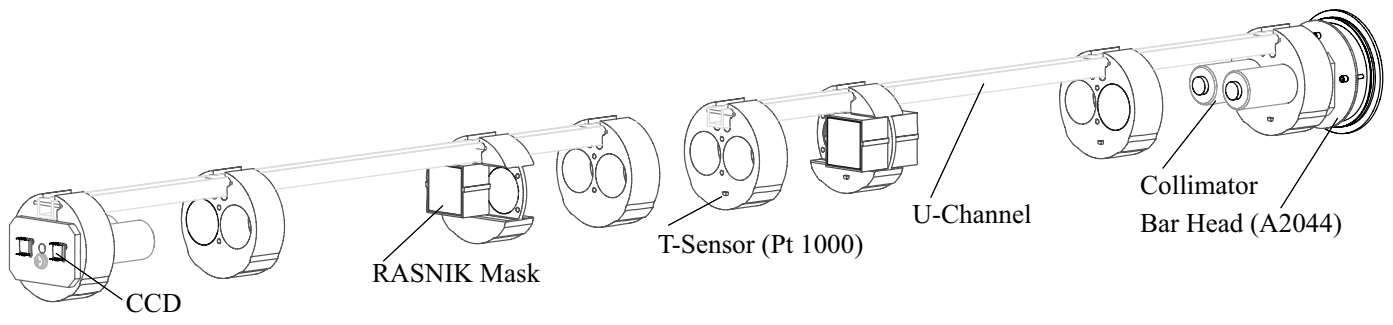

Figure 8: Inner parts of an alignment bar with four RASNIKs. The two units are inserted from either end of the alignment bar.

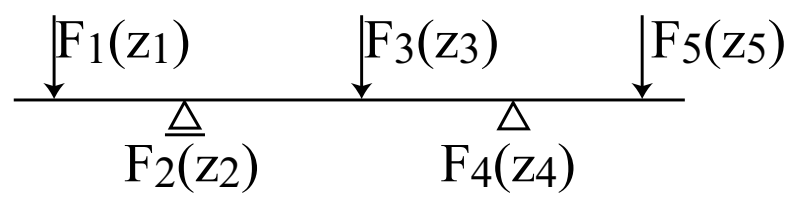

Figure 9: Cylindrical tube supported in two points and exposed to three external forces $F_{1}, F_{3}$, $F_{5}$; the two forces $F_{2}, F_{4}$ respond in the support points. 

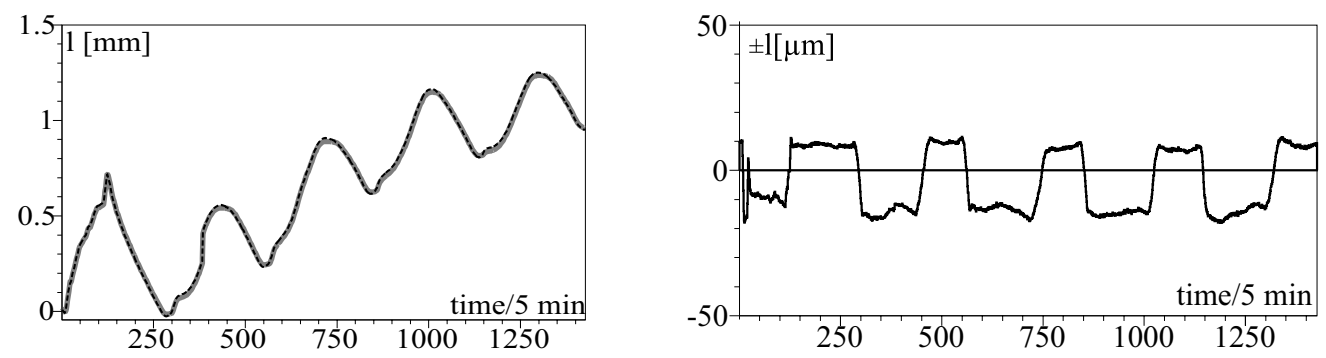

Figure 10: Change in bar length over 4.5 days in an ambient temperature environment ranging from $18^{\circ} \mathrm{C}$ to $27^{\circ} \mathrm{C}$; Left: calculated from temperature sensor measurements and measured with quartz rod and capacitive sensor; Right: difference between the two.
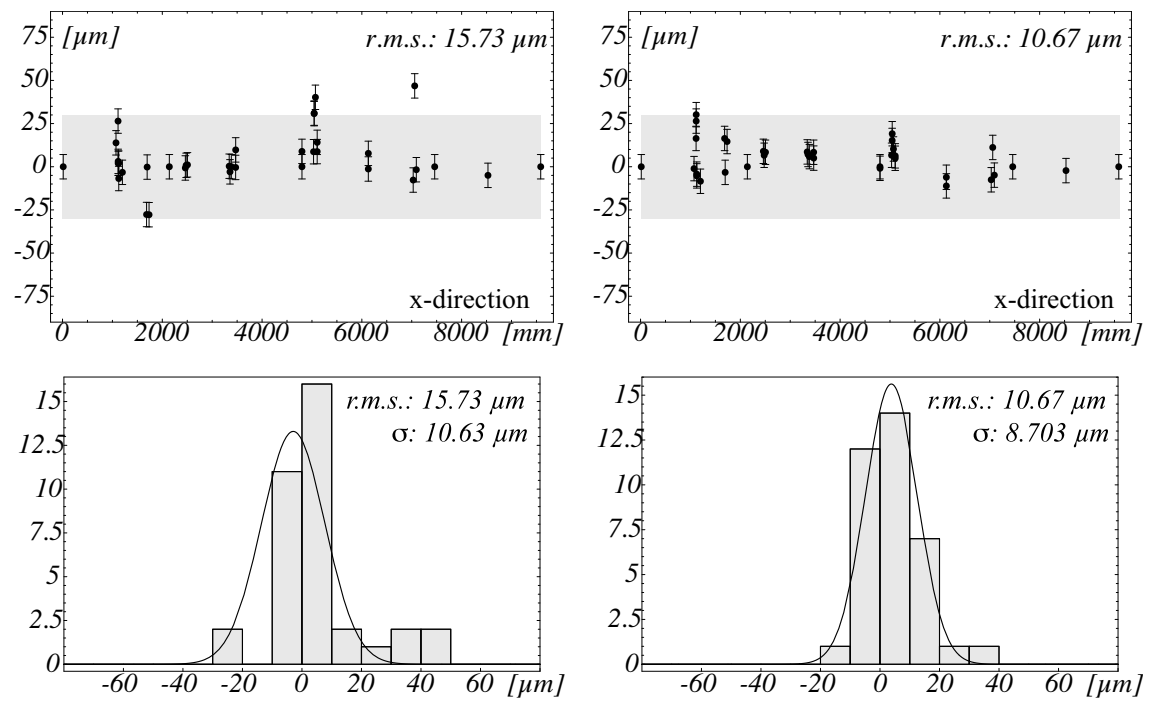

Figure 11: Ball positions predicted from the $0^{\circ}$ position, subtracted from the measurements in the $180^{\circ}$ position, for the two coordinates transverse to the bar axis. 

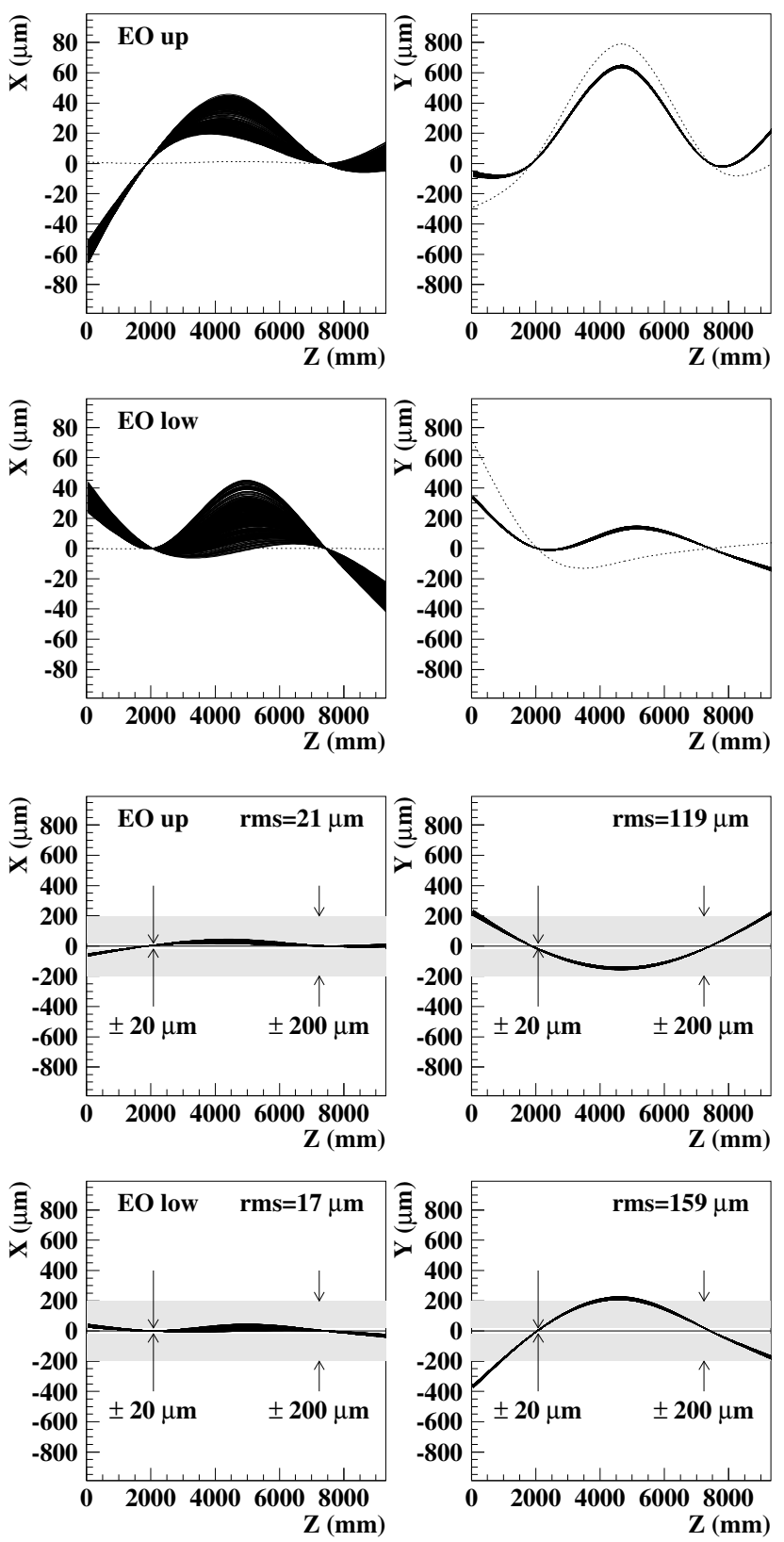

Figure 12: Top: measured deformations of two of the alignment bars in the test setup relative to their initial shape during calibration (which corresponds to $X=Y=0$ ). The $X$ coordinate is approximately horizontal, $Y$ is approximately in the vertical plane, and $Z$ is the bar axis. The bar named 'EO-up' was installed inclined by $45^{\circ}$ in the setup, the one named 'EO-low' was horizontal. The bands of black lines represent variations of the deformation during temperature changes up to $5^{\circ} \mathrm{C}$, the dotted curve the expected deformation (i.e. taking into account the known forces acting on the bar, before applying the final correction with three effective forces). Note the different scales of the vertical axes. Bottom: difference between measured and expected deformations. Arrows indicate the design uncertainty of the bar shape of $20 \mu \mathrm{m}$, and the acceptable difference between expected and observed deformation of $200 \mu \mathrm{m}$ for an assumed interpolation error of $10 \%$. 
H8 setup
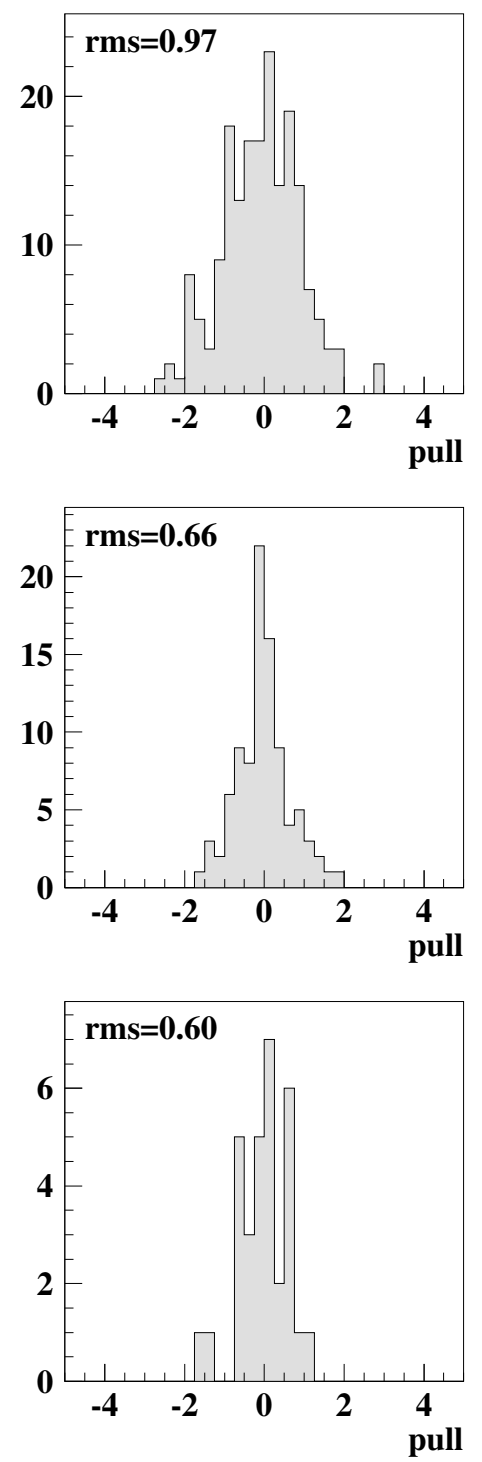

simulation (300 setups)
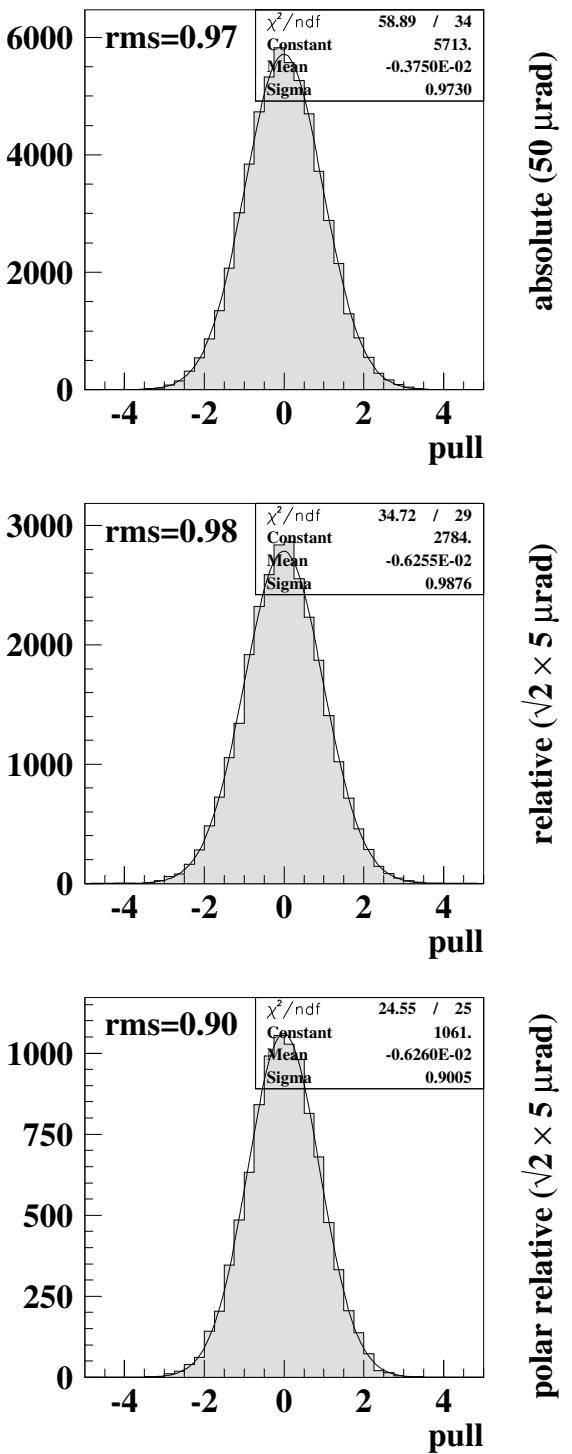

Figure 13: Pull values for all BCAMs in the setup after constraining the alignment bars to the locations given by the survey. Left: real data from the setup. Right: simulated data (from 300 simulated setups). Top: BCAM measuring the absolute position of one light source on its partner ( $X$ and $Y$ combined in one histogram). Centre: BCAM measuring the separation of the two light sources on its partner. Bottom: polar BCAM measuring the separation of two light sources on its two partners. Note that plots of real data reflect a single setup, while plots of simulated data show the average distribution for many setups; the entries in plots of real data should therefore approximate a Gaussian distribution only in the limit of many sensors (which is not reached in H8). The r.m.s. values have been computed from the histograms and assuming zero mean, i.e. they reflect the sum in quadrature of mean and width. The error of the r.m.s. values from real data is 0.10 for the top and middle plots, and 0.18 for the bottom plot; the error of those from simulation is negligible. 

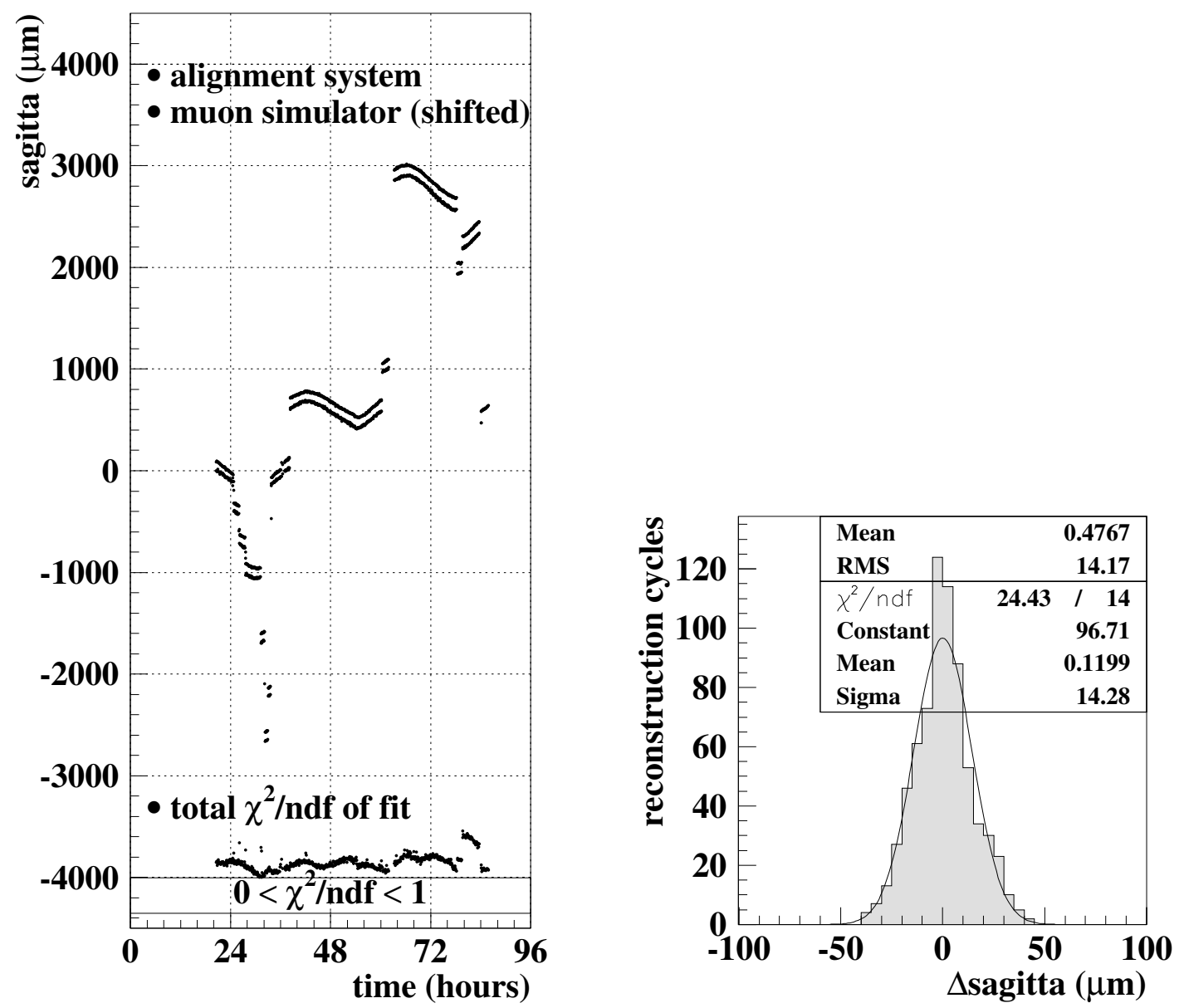

Figure 14: Left: sagitta variations reconstructed from the alignment system (upper points) and measured by the muon simulator (lower points, shifted by $100 \mu \mathrm{m}$ for clarity of presentation), over a period of 2.5 days. Smooth variations are temperature-induced, steps come from chamber shifts and rotations. The total $\chi^{2} /$ ndf of the alignment fit is shown at the bottom; the horizontal lines indicates the range $0<\chi^{2} /$ ndf $<1$. Right: sagitta difference between alignment system and muon simulator. 